\title{
Scott G. Chaplowe and J. Bradley Cousins. (2016). Monitoring and Evaluation Training: A Systematic Approach. Thousand Oaks, CA: SAGE. Hardcover, 439 pages. (ISBN 978-1-4522-8891-8)
}

\section{Reviewed by Michele Tarsilla, UNICEF, West and Central Africa, Regional Office, Dakar, Senegal}

When I first looked at the title of this book, I confess that I had mixed feelings. First, the book's focus on "monitoring and evaluation" seemed a bit too broad: monitoring and evaluation certainly complement each other; however, their respective specificities risk being neglected when the " $\mathrm{M}$ " and the "E" are bundled together. Next, the book's focus on "training" seemed too narrow to tackle the complexity of the M\&E capacity realms. Specialized literature confirms that training by itself is only one of the many strategies one could pursue to strengthen $M \& E$ capacity. I have argued for years that the excessive attention given to training (a phenomenon that I refer to as the objectification of capacity) has undermined efforts made thus far to strengthen national M\&E capacity. To the contrary, it is through interventions and strategies geared toward capacity strengthening at not only the individual but also the organizational and systemic levels that sustainable and contextually relevant Evaluation Capacity Development (ECD) occurs (Tarsilla, 2014).

Two factors motivated me to delve into this book despite an initial skepticism. First was my familiarity with and appreciation of the co-author's prior work (Bradley Cousins is a professor at the University of Ottawa and a prolific author of articles on capacity building and participatory approaches; Scott Chaplowe is a senior evaluation specialist with an extensive range of experience both within and outside of numerous international organizations). Second, the "systematic approach" referred to in the subtitle set this resource apart from the most M\&E training manuals available on the market, which often jump directly into Kirkpatrick's (1996) model and a few facilitation tricks to get workshop participants learning about M\&E.

Fortunately, some of my earlier doubts dissipated rapidly once I started reading the book. Right from the introduction, I was positively surprised by the authors' continued reference to the need for "systemic thinking" when planning, designing, and developing training events. Likewise, I enjoyed the insightful presentation of the adult learning principles, different types of learning, and key trainers' competencies. Page after page, the book made me reflect upon my own 
practice and spurred some new ideas about how I could enhance the quality of my training planning in the future. However, in doing so, I also realized that the book is indeed quite dense (a total of 405 pages distributed across 11 chapters, and one supplementary section including 99 real-life examples of capacity building) and that the less-experienced reader may feel a bit intimidated by the vast quantity of information provided. That notwithstanding, the book is very well written (I read it cover to cover over a weekend) and logically structured: each chapter starts with clearly articulated objectives and key definitions ("learning transfer" and "utility" are explained quite cogently) and ends with a critical summary and a list of recommended technical resources for future consultation.

The book is divided into three parts. Part 1 ("Key Concepts for M\&E Training") provides readers with an understanding of the foundational elements of M\&E training and clarifies different elements and types of impactful training (from formal to non-formal training; from informal to incidental). I especially appreciated the focus of Chapter 1 on how to enhance training usefulness, often referred to as "training transfer," as well as of Chapter 3, dedicated to the need to design contextually relevant training programs, as training is not isolated from, and requires a synchronization with, trainees' organizational culture as well as the external environment with which those organizations interact. Likewise, I found Chapter 4 on adult learning particularly instructive: it provided a solid theoretical grounding for some of the most influential conceptual frameworks in the training field: from Knowles's six assumptions of learning to andragogy and the taxonomy of learning styles (i.e., activists, reflectors, theorists, and pragmatists). Chapter 5 is also quite unique in that it discusses the six trainer key competencies. The only downside is that the book as a whole relates mostly to mainstream M\&E and not does not explain how training could be adapted when used in relatively new evaluation domains, such as developmental evaluation, or more complex environments, such as public policy evaluation training.

Part 2 ("A Systematic Approach to M\&E Training") is more practical in scope and spells out the different phases of the ADDIE framework (training analysis, design, development, implementation, and evaluation). The checklist at the end of Chapter 6 on actions required to be taken during each phase of the framework is particularly interesting, as it helps give structure to a process that, due to the large number of parties involved, is often scattered and/or not well sequenced. Chapter 7 on needs and outcomes analysis is quite useful too, as it suggests the use not only of "backward design" (Wiggins \& McTighe, 2005) during the identification and verification of training outcomes, but also of trainee analysis coupled with task and gap analysis, as a way to better enhance the training utility. Likewise, I found Chapter 8 on the selection of learning objectives quite interesting, especially the section discussing the necessary linkages among performance, condition, and criterion, although it is rather detailed in places. In real circumstances, a trainer would hardly include in a training plan all of the following: training outcomes, learning goal, primary learning objective, and secondary learning objectives. Furthermore, Chapter 10 stands out for its provision 
of tips on how to handle and successfully eliminate disruptive behaviour during the training time. Lastly, Chapter 11 skillfully covers the different approaches to evaluating training programs.

Part 3 ("M\&E Training Methods and Techniques") presents the reader with a plethora of techniques (99 in total) that are expected to enrich the quality and effectiveness of M\&E training (e.g., from learning games and practicum experiences to role-playing and M\&E software activities). Details for each of these tools, including a balanced discussion of related advantages and disadvantages, are particularly useful; however, the authors could have discussed in greater depth the implications of the use of some of these techniques during online training deliveries, as this section is focused mostly on face-to-face M\&E training. Even with these caveats, and although some techniques replicate those found in other manuals, I am likely to adopt the more inventive exercises discussed, including popcorn introductions and group résumés, for helping break the ice among training participants and creating a bond among them from the beginning of the training program: the "if ... then" exercise for helping trainees develop more logical and evidence-based theories of change for their respective clients and organizations; the WEM3 exercise for getting trainees used to taking different-and somewhat diverging-perspectives into account during the planning, conduct, and dissemination of evaluations; and the participant-driven case studies and the fishbowl simulations for anchoring the training to the participants' life practice and enhancing the quality of evaluation peer review.

Overall, this book represents an ideal addition to the "toolbox" of (i) training professionals with a sufficient knowledge of M\&E concepts, (ii) M\&E training managers, (iii) educators and students in post-secondary and continuing contexts, and (iv) training of trainers (ToT) facilitators. Despite not being able to tackle the issues of M\&E capacity development at a more systemic level, this book has the merit of bringing rigour and intentionality back into the planning, design, and delivery of M\&E training, while also providing a rich theoretical and sound experiential base.

\section{REFERENCES}

Kirkpatrick, D. (1996). Revisiting Kirkpatrick's four-level-model. Training \& Development, $1,54-57$.

Tarsilla, M. (2014). Evaluation capacity development in Africa: Current landscape of international partners' initiatives, lessons learned and the way forward. African Evaluation Journal, 2(1), Art. \#89, 13 pages. https://doi.org/10.4102/aej.v2i1.89

Wiggins, G., \& McTighe, J. (2005). Understanding by design. Alexandria, VA: Association for Supervision and Curriculum Development (ASCD). 